\title{
Treatment of Chronic Venous Ulcers Using New Four Layers Compressive Bandage Dressing
}

\author{
Kaushal K Tiwari, ${ }^{1}$ Krishna G Shrestha, ${ }^{1}$ Bijay Sah, ${ }^{1}$ D. Jaypal Reddy' \\ 'Department of Cardiothoracic and Vascular Surgery, College of Medical Sciences, Teaching Hospital, Bharatpur, Chitwan, \\ Nepal.
}

\section{ABSTRACT}

Introduction: Lower-extremity ulcers represent the largest group of ulcers presenting to an outpatient department. It is a cumbersome, difficult to treat disease, which causes high morbidity and huge cost for the patient and healthcare system. Current standard treatment includes compression therapy. However, majority of patients need long term treatment with minimal efficacy. Aim of our study is to evaluate efficacy of four layers compressive bandages for the management of chronic venous ulcers.

Methods: In Group A, we have prospectively included 20 patients with chronic venous ulcers on lower limbs for four layers hosiery bandage using Velfour bandage. Other 15 patients, Group B, were treated with conventional wound dressing. Velfour and crepe bandage were done once weekly for three weeks.

Results: DVT was cause of chronic venous ulcer in 70\% patient in group A and in 73.3\% in Group B. Majority of patients were having left sided chronic venous ulcers. The mean duration of the ulcers was 15.6 vs 10.86 months (group A vs. group B). At the end of $3^{\text {rd }}$ week, in 55\% wounds in Group A were healed except few big and deep wounds remained. Most of these wounds also became smaller with minimal discharge. Size of wounds significantly decreased in Group A vs. Group B patients $(0.7 \pm 0.81$ $\mathrm{cm}$ vs. $1.73 \pm 0.77 \mathrm{~cm}, \mathrm{p}<0.00031)$. However, cost of treatment in group A remained higher than group B.

Conclusions: Our study has shown that four layer compressive bandage using Velfour is an easy, effective, and reproducible method of treatment for the chronic venous ulcer.

Keywords: compression bandage; treatment; venous ulcer.

\section{INTRODUCTION}

Lower-extremity ulcers represent the largest group of ulcers presenting to an outpatient and inpatient. Ulcers due to venous insufficiency and venous hypertension make up the largest subgroup of these ulcers. Such chronic leg ulceration represents an advanced clinical manifestation of venous insufficiency. Venous ulcers is defined by the American Venous Forum as "a full-thickness defect of skin, most frequently in the ankle region, that fails to heal spontaneously and is sustained by chronic venous disease, based on venous duplex ultrasound testing. "1 It is cumbersome, difficult to treat disease, which causes high morbidity and huge cost for

Correspondence: Dr. Kaushal K. Tiwari, Department of Cardiothoracic and Vascular Surgery, College of Medical Sciences, Teaching Hospital, Bharatpur-10, Chitwan, Nepal. E-mail: drkaushalkł@ yahoo.com, Phone: +977-9801059361. 
the patient and healthcare system. About 4-4.5 million people are affect and $2-3 \%$ of healthcare budget has spent in the United States for the treatment of chronic venous ulcers.

There has been considerable progress in our understanding of the pathophysiology, hemodynamics, venous imaging, and therapeutic options for venous ulcers, including endovenous ablation, iliac vein stenting, and veinvalve repair techniques. ${ }^{2}$ Nevertheless, current standard treatment includes local wound care with the application of compression. ${ }^{3,4}$ Furthermore, interventions for chronic venous ulcers have evolved to painless, minimally invasive, office-based procedures performed under local anesthesia. Recent advances in the endovascular management of lower-extremity superficial venous insufficiency have the potential to enhance initial and long-term management of these patients, as minimally invasive procedures provide faster recoveries and fewer procedural risks. Early intervention for venous insufficiency has shown a significant decrease in long-term ulcer recurrence rates, and may increase healing of venous ulcers as well. ${ }^{5}$ However, such sophisticated treatment options are not easily available everywhere, especially in the developing countries. Therefore, an easily available, reproducible and effective treatment option is necessary to treat this chronic problem.

Aim of our study is to evaluate efficacy of four layers compressive bandage for the management of chronic venous ulcers. Four layers compressive bandage consists of first layer of orthopedic wool, followed by cotton conforming bandage, elastic compression bandage and uppermost layer is cohesive compression bandage (Figure1).

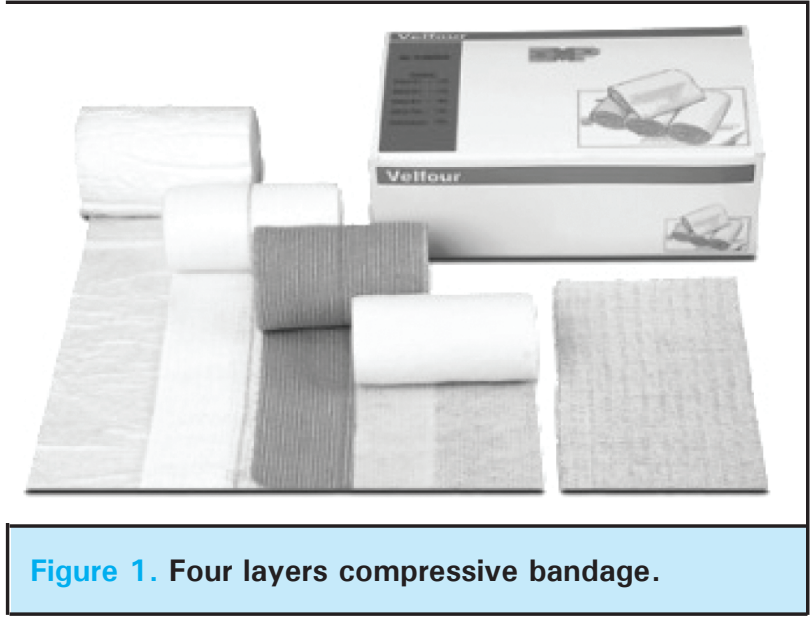

\section{METHODS}

From June 2014 to until May 2015, thirty-five patients treated in the department of CTVS, college of Medical Sciences, Teaching hospital, Bharatpur with diagnosis of chronic venous ulcers secondary to varicose vein and/ or DVT. Written consent from every patient taken prior to the enrollment in the study. Institutional ethical and review committee has approved the study. Out of these patients, in Group A, we have prospectively included 20 patients with chronic venous ulcers on lower limbs of different severity for four layers compressive bandage using Velfour (Datt Mediproducts Ltd, New Delhi, India) bandage kits. Other 15 patients, Group B, treated with conventional wound dressing and crepe bandage due to lack of money or other factors contraindicating the use of four layers compressive bandage; i.e. patients with significant arterial disease, ABPI $<0.8$ and patients with decompensated heart failure. Velfour bandage was applied once weekly for 3-4 weeks consecutively. While conventional dressing was done alternate day for 3-4 weeks using local antiseptics and single layer Crepe bandage or elastic stockings. Debridement of the wounds done wherever it required. Both group of patients received limb elevation, anticoagulation, antibiotics, and other supportive treatment as per requirements.

\section{RESULTS}

Table 1. General characteristics of the patients are presented.

\begin{tabular}{|llll|}
\hline SN & Variables & \multicolumn{2}{c|}{ Values } \\
& & Group A & \multicolumn{1}{c|}{ Group B } \\
1 & Age & $50.8 \pm 17.83$ & $45.07 \pm 15.07$ \\
2 & Male & 12 patients & 8 patients \\
3 & Female & 8 patients & 7 patients \\
4 & Hypertension & 9 patients & 8 patients \\
5 & Diabetes & 9 patients & 6 patients \\
6 & Obesity & 3 patients & 3 patients \\
7 & DVT & 14 patients & 11 patients \\
8 & Varicose vein & 10 patients & 7 patients \\
\hline
\end{tabular}

General characteristics of the patients enrolled in both groups were identical. Mean age of patients was $50.08 \pm 17.83$ in Group $A$ and $45.07 \pm 15.07$ in Group $B$ (Figure 2). There were 12 and 8 males in group $A$ and $B$ respectively (Figure 3 ). 


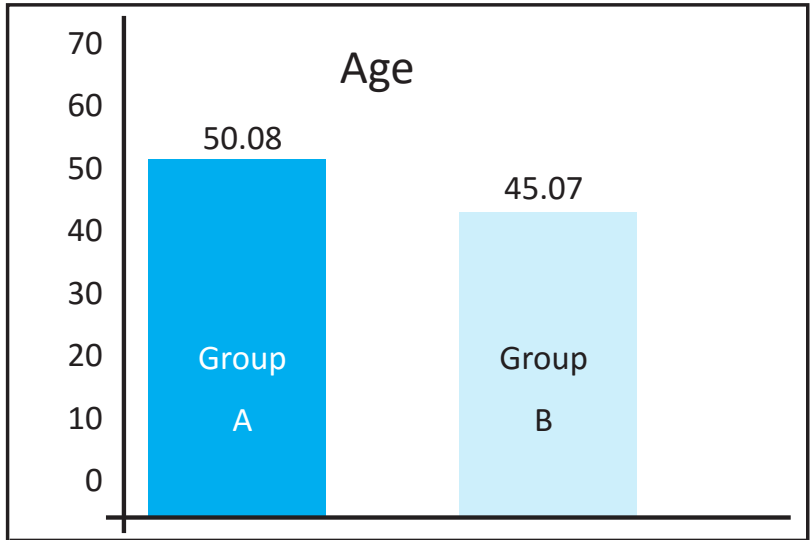

Figure 2. General characteristics of the patients age.

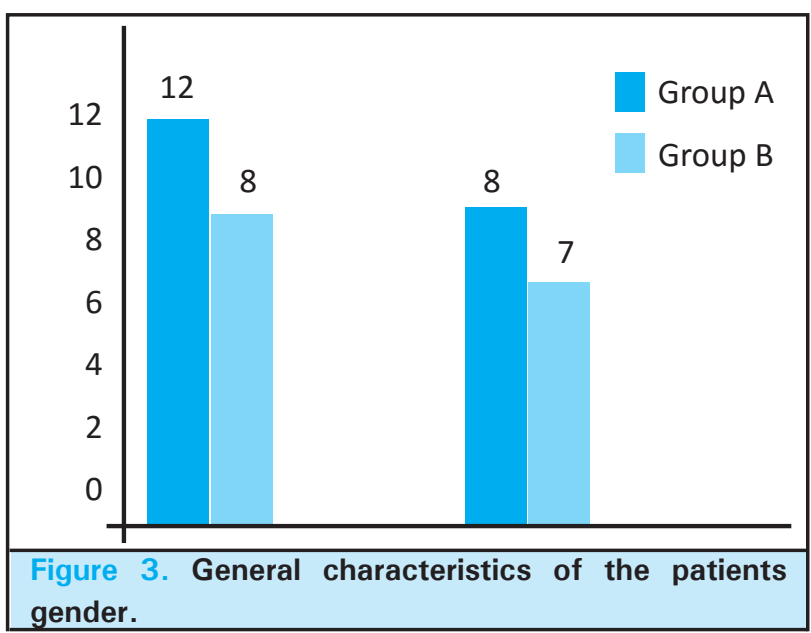

Forty five percent (9 patients) were suffering from diabetes mellitus in Group A, while 40\% (6 patients) in Group B. There were no significant difference in the HTN in both group (45\% vs. 53.3\%, Group A vs. Group B) (Figure 4). Causes of chronic venous ulcers were varicose vein in 50\% (10 patients) in Group $A$ and in $46.7 \%$ (7 patients) in Group B (Figure 4). While, DVT was cause of chronic venous ulcer in $70 \%$ (14 patients) of group $A$ and in $73.3 \%$ (11 patients) of Group B population (Figure 5).

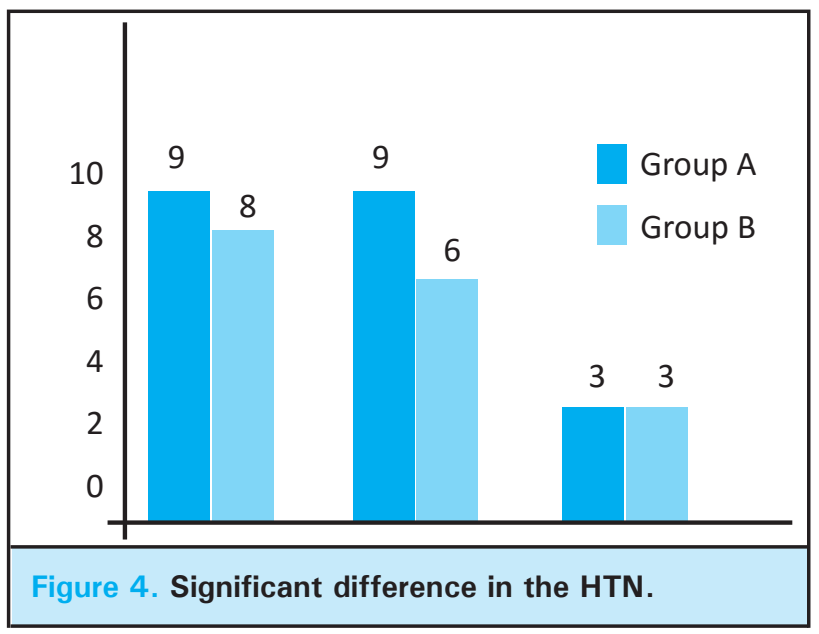

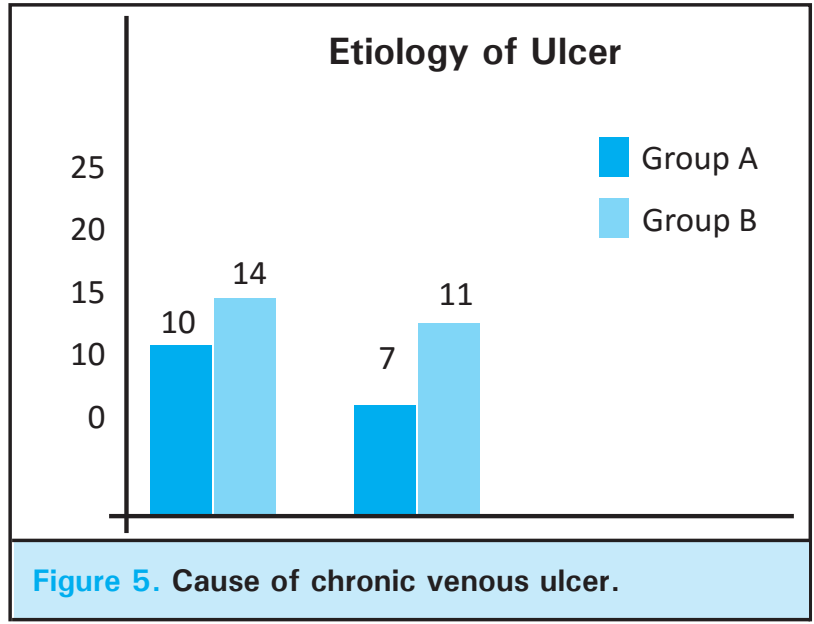

Majority of patients were having left sided chronic venous ulcers in both the groups $165 \%$ in Group A, $66.7 \%$ in Group B). Preoperative size of wounds were similar in both groups $(3.2 \pm 0.92 \mathrm{~cm}$ in Group A, $3.06 \pm 0.94 \mathrm{~cm}$ in Group B) (Figure 6). The mean duration of the ulcers was 15.6 months in Group $A$ and 10.86 months in Group B (Figure 7).
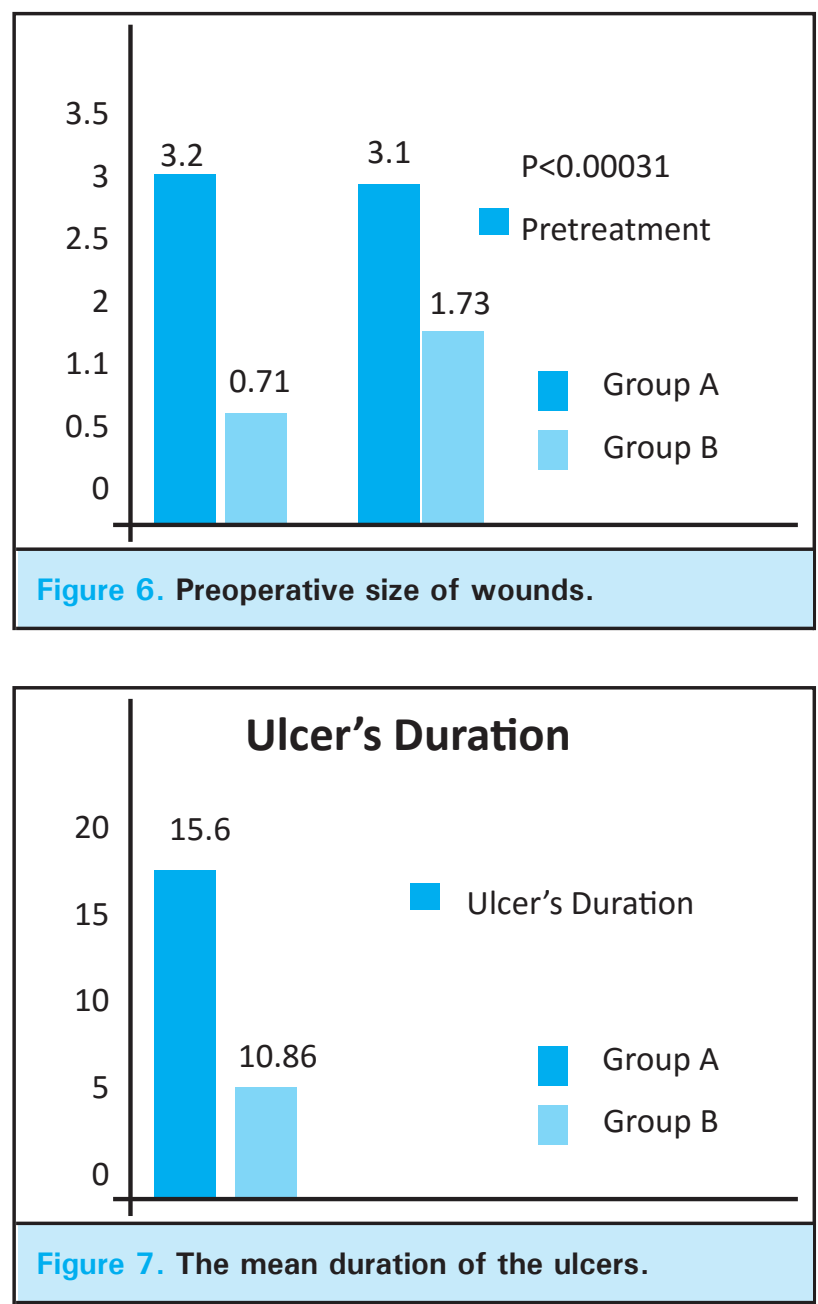
Sixty five percent (13 patients) of Group $A$ and sixty percent (9 patients) of Group B had history of recurrent ulcers. Average hospital stay was similar in both groups (5.75 days vs. 5.53 days) (Figure 8). In Group A patients Velfour compression bandage was applied for a mean period of 3.12 weeks, while in Group B Crepe bandage applied for a mean period of 3.33 weeks (Figure 9).
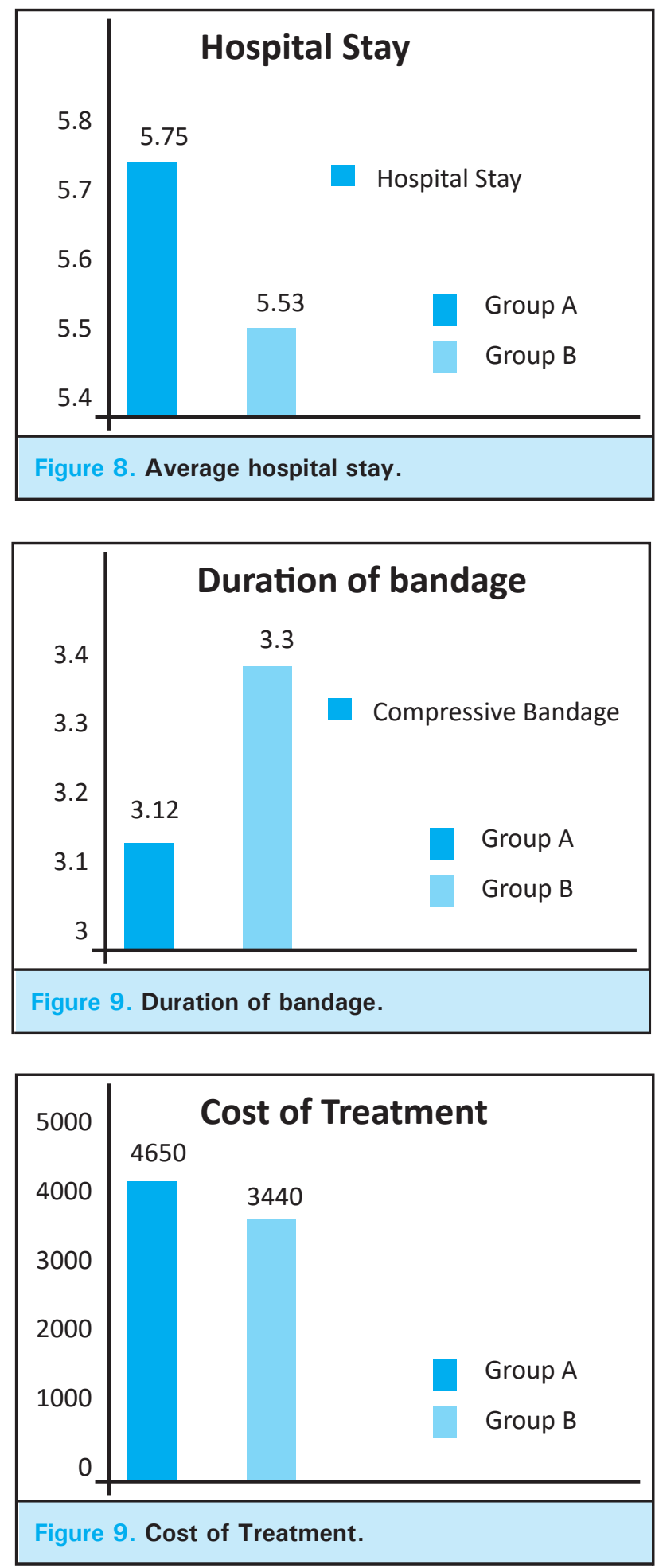

In Group A, on second week, size of the wounds became significantly smaller, wound was cleaner and sign of healing noted. At the end of $3^{\text {rd }}$ week, in $55 \%$ (11 patients), wounds in Group A were healed except few big and deep wounds remained, but those wounds were cleaner with good granulation tissue. Most of these wounds also became smaller with minimal discharge. Size of wounds significantly decreased in Group $A$ vs. Group B patients $(0.7 \pm 0.81 \mathrm{~cm}$ vs. $1.73 \pm 0.77$ $\mathrm{cm}, \mathrm{p}<0.00031$ ) (Figure 5). In contrast, group $B$ patients showed slower wound healing, required more dressing and at the end of $3^{\text {rd }}$ week, about $80 \% \quad(12$ patients) had still infected, non-healing wound with continuous discharge. However, cost of treatment in Group A remained significantly higher than group $B$ $(4650 \pm 1451.8$ vs. $3440 \pm 792.64$ Nepalese Rupees, $\mathrm{p}<0.006$ ) (Figure 9).

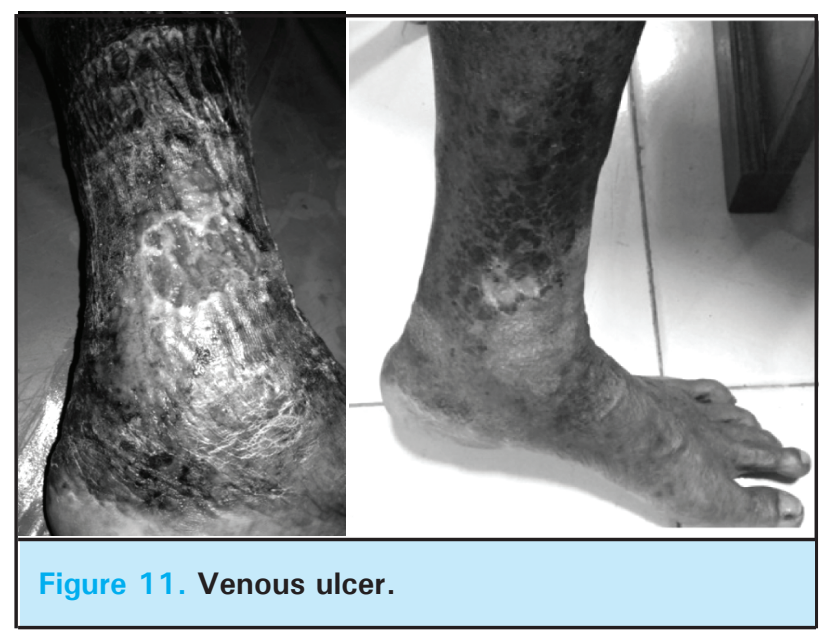

\section{DISCUSSION}

Venous ulcer is the most serious consequence of chronic venous insufficiency. This is a common and often disabling condition. Due to their frequency and chronicity, venous ulcers have a high socioeconomic impact, with treatment costs accounting for $3-4 \%$ of the health care budget in Western countries. The disease is known for more than 3.5 millennia with wound care centers established as early as 1500 BC. Unfortunately, still today, it is a very poorly managed medical condition despite that a great deal has learned about the pathogenesis and treatment for venous ulcerations. It is observed that many physicians treat the wound and not the cause of the problem. ${ }^{6}$ The recurrent nature of venous ulcers underscore the need for treatment of the underlying pathophysiology, that is, ambulatory venous hypertension produced by venous valve reflux alone or in conjunction with venous obstruction. ${ }^{7}$ Incompetent veins or valves or impaired muscle function may lead to abnormal calf muscle pump function that can elevate 
ambulatory venous pressure (venous hypertension). This hypertension subsequently results in local venous dilatation and pooling, concomitantly trapping leukocytes that may release proteolytic enzymes that destroy tissues. Venous pooling also induces interendothelial pore widening and deposition of fibrin and other macromolecules that "trap" growth factors within them, rendering them unavailable for wound repair. ${ }^{8}$

The evaluation of patients with venous ulcers should include a thorough medical history for prior deep venous thrombosis, assessment for hypercoagulable state, and a physical examination. In the presence of venous insufficiency, lifestyle modification is required with affected individuals performing daily intervals of leg elevation to control edema; use of elastic compression garments; and moderate physical activity, such as walking wearing below-knee elastic stockings. Meticulous skin care, treatment of dermatitis, and prompt treatment of cellulitis are important aspects of medical management. ${ }^{9}$ Patient comfort and satisfaction with both compression therapy and wound care are critical to the success of venous leg ulcer treatment.

Compression therapy with crepe bandage or elastic stockings are commonly used methods of treatment for the venous insufficiency and venous ulcers. Compression therapy is directed at counteracting the chronic inflammation in the tissues and at decreasing ambulatory venous hypertension in the area. Compression therapy helps to decrease the venous hypertension and aids healing. Compression therapy, the mainstay treatment, reduces edema, reverses venous hypertension, and improves calf muscle pumping function. ${ }^{10}$ Topical agents may be used to help decrease the bacterial load in the wound, provide a moist healing environment for dry wounds, or absorb the exudate in wounds with a lot of drainage. Orthopedic wool and cotton conforming layer of Velfour bandage have sufficient absorption capacity to manage exudate for up to a full week without needing re-application. Interventions to decrease the ambulatory venous hypertension can help healing ulcers in patients with either active or chronic ulcers. Velfour ${ }^{\mathrm{TM}}$ Four-layer bandage creates effective compression by delivering approximately $40 \mathrm{mmHg}$ of pressure at the ankle, reducing to $17 \mathrm{mmHg}$ at the knee. In addition, these four layers compression bandage maintain graduated, sustained and effective compression for a longer duration even up to a week after application, which is not possible with Crepe bandage alone. Extended wear time allow satisfactory drainage of the venous pool, reducing local venous hypertension. Additionally, keeping these compression bandages for one week improves patient's comfort and compliance as there is no need for repeated dressing and OPD visits. In Group A patients significant improvement in the wound healing is the result of decreased venous hypertension and venous pooling for a continuous and longer period. None of the patient in Group A complained of any discomfort and impairment with mobility using these four layers compressive bandages. While, patients with Crepe bandage had common complain of loosening up of the bandage and falling down during mobility. It causes discomfort and non-compliance in many cases. Cost of treatment in the Group A patient was significantly higher than Group B. However, if we take into account the loss of working hours due to regular OPD visits, physical exhaustion, social isolation and psychosocial trauma to the patient and their caretaker, overall cost in Group B could be higher than Group A. Two layer compression hosiery is also compared with four layer compressive bandage in Venous Leg Ulcer Study IV, VenUS IV. In this randomized study, Ashby et al has shown that Two-layer compression hosiery is a viable alternative to the four-layer bandage-it is equally as effective at healing venous leg ulcers. However, a higher rate of treatment changes in participants in the hosiery group than in the bandage group suggests that hosiery might not be suitable for all patients. ${ }^{11}$

\section{CONCLUSIONS}

Based on our findings, we conclude that Compression bandage remain the Gold standard treatment of chronic venous ulcer in addition to the local and systemic treatment of the cause. Moreover, four layers compressive bandage is superior to Crepe bandage/ stockings in treating these cumbersome ulcers. Velfour helps to heal chronic venous wounds faster and effectively. It is easy to apply and can be repeatedly used without any difficulties.

Limitation of our study is small number of study population and non-randomization of the cases. In some cases, it might create bias in the result. However, this being the initial study, it can be taken as a pilot study and in future a randomized study with higher number of patient can be enrolled to support this conclusion.

\section{REFERENCES}

1. Abbade LP, Lastória S. Venous ulcer: epidemiology, physiopathology, diagnosis and treatment. Int J Dermatol. 2005; 44: 449-456.
2. Verma H, Tripathi RK. Algorithm-based approach to management of venous leg ulceration. Sem Vasc Surg 2015 Mar;28(1):54-60. 
3. Romano F, Paolino FM, Rizzo BA, Russo A et al. The use of growth factors, CD34+ cells and fibrin for the management of chronic venous ulcers. Int Wound J 2015 Sep 15. Doi 10.1111/iwj.12500.

4. Powell G, Wicks G, Wilk K. Managing venous leg ulcers using compression therapy and dressings. Br J Nurs. 2015 Aug;12;14 Suppl 15: S42-S49.

5. istner RL, Shafritz R, Stark KR, Warriner RA $3^{\text {rd }}$. Emerging treatment options for venous ulceration in today's wound care practice. Ostomy Wound Manage . 2010 Apr 1;56(4):E1-11.

6. Word R. Medical and surgical therapy for advance venous insufficiency. Surg Clin North Am. 2010 Dec;90(6).1195-2014.

7. Lal BK. Venous ulcers of the lower extremities: Definition, epidemiology, and economic and social burden. 2015 Mar; 28(1):3-5.
8. Trent JT, Falabella A, Eaglestein WH, Kirsner RS. Venous ulcers: pathophysiology and treatment options. Ostomy Wound Mamage. 2005 May;51(5):38.55.

9. Pascarella L, Shortella CK. Medical management of venous ulcers. Semin Vasc Surg. 2015 Mar;28(1):21-28.

10. Frykberg RG, Banks J. Challenges in the treatment of chronic wounds. Advances in wound care. 2015, Vol.4, No. 9; 560-582.

11. Ashby RL, Gabe R, Ali S, Adderley U, Bland JM, Cullum NA, Dumville JC, Iglesias CP, Kang'ombe AR, Soares MO, Stubbs NC, Torgerson DJ. Clinical and cost-effectiveness of compression hosiery versus compression bandages in treatment of venous leg ulcers (Venous leg Ulcer Study IV, VenUS IV): a randomised controlled trial. Lancet. 2014 Mar8:383(9920):871-879. 